\title{
Wide control of the group velocity of light in optical fibers
}

\author{
Miguel González Herráez*, Kwang-Yong Song ${ }^{\dagger}$ and Luc Thévenaz \\ Nanophotonics and Metrology Lab. Ecole Polytechnique Federale de Lausanne, CH-1015, Switzerland \\ E-mail: miguel.gonzalez@epfl.ch. * On leave from Dept. of Electronics, Univ of Alcalá, Madrid, Spain. \\ ${ }^{t}$ Presently with the Dept. of Electronic Engineering, Univ. of Tokyo, Japan.
}

\begin{abstract}
We demonstrate experimentally that a wide control of the group velocity of light can be achieved in conventional optical fibers by use of the stimulated Brillouin scattering effect. Zero, negative and large positive group indices can be readily achieved with a simple experiment. (C) 2005 Optical Society of America

OCIS codes: (290.5900) Scattering, stimulated Brillouin; (060.4370) Nonlinear optics, fibers; (999.9999) Slow light;
\end{abstract}

\section{Introduction}

Recent experiments have demonstrated the possibility to achieve a wide group delay control in optical fibers by use of the stimulated Brillouin scattering (SBS) effect [1,2]. Despite its recent demonstration, this technique is already thought to offer a vast potential for performing all-optical packet routing and random access memories. Up to now, slow and fast light experiments in fibers using SBS have concentrated on achieving large fractional group delays in optical pulses, but with modest changes in the group velocity achieved in the fiber $[1,2,3]$. In this paper we demonstrate that a wide control of the group velocity of optical signals propagating in fibers can be readily achieved with conventional instrumentation. The extreme case of a negative group velocity is also demonstrated.

\section{Theory}

In all the experiments of slow and fast light, the presence of narrowband spectral resonances is required. Spectral resonances have a complex response function, so that they introduce an extremely narrowband peak in the absorption/gain characteristics of the medium while there is also a sharp transition in the effective refractive index of the material. This sharp transition induces a strong change in the group index, which is responsible for large changes in the relative delay of an optical pulse as it travels through a material. In our case, we use the narrowband gain and loss mechanisms of SBS to achieve strong changes in the group index of optical pulses [1].

Figure 1(a) shows the relation between the Brillouin gain, the phase index change $(\Delta n)$ and the resultant group index change $\left(\Delta n_{g}\right)$ generated by SBS in an optical fiber. The process of SBS is usually described as the interaction of two counter propagating waves, a strong pump wave and a weak probe wave. If a particular phase matching condition is satisfied (namely $f_{\text {Pump }}=f_{\text {probe }}+v_{B}, v_{B}$ being the Brillouin frequency), an acoustic wave is generated. This acoustic wave scatters photons from the pump to the probe wave, stimulating the process.

From a practical point of view, the SBS can be viewed as a narrowband amplification process, in which a strong pump wave produces a narrowband $(30-50 \mathrm{MHz})$ gain in a spectral region around $f_{\text {Pump }}-\nu_{B}$ and a loss around $f_{\text {Pump }}+v_{B}$. As observed in any sharp spectral transition, a refractive index change is associated with the Brillouin gain/loss process and a substantial change of the group index $n_{g}=n+\omega d n / d \omega$ is acquired as a result of the sharp index transition as shown in Fig 1(a).

For a gain process, the group index change $\left(\Delta n_{g}\right)$ is positive resulting in slow light, while a loss gives a negative $\Delta n_{g}$ corresponding to the case of fast light. In this latter case extreme situations may lead to $n_{g}<1$, that is a signal envelope propagating faster than the vacuum light velocity $c$, or even to a negative group velocity (the peak of the pulse exits the fiber before it enters). The fact that such signals nevertheless do not break the principles of causality and relativity has been thoroughly discussed [4]. 


\section{WC3}

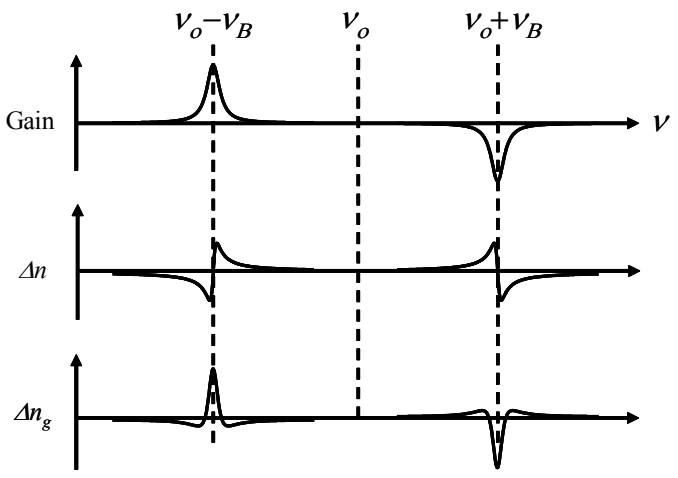

(a)

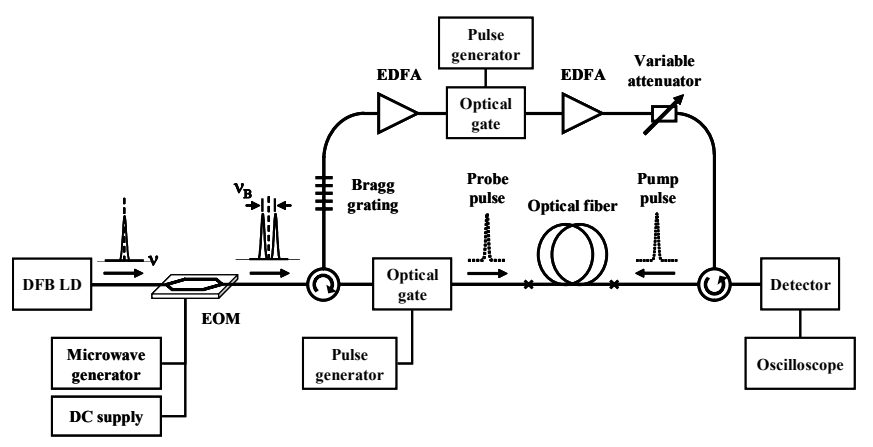

(b)

Fig. 1. (a) Gain, refractive index change $(\Delta n)$ and group index change $\left(\Delta n_{g}\right)$ generated by stimulated Brillouin scattering in an optical fiber. $v_{o}$ and $v_{B}$ are the optical frequency of the Brillouin pump and Brillouin shift of the fiber, respectively. A probe wave propagating in the opposite direction experiences a narrowband gain if its frequency is $v_{0}-v_{B}$ and a narrowband loss if its frequency is $v_{0}+v_{B}$. (b) Experiment for the observation of pulse advancement and delaying using SBS in optical fibers.

\section{Experiment}

Figure 1(b) shows the experimental configuration, which is basically similar to that given in [1] except for a pulse-operation of a Brillouin pump wave. Since the time delay (advancement) of the probe pulse only depends on the overall gain (loss) experienced along the full fiber length for a given type of fiber [1], one can increase the group index change drastically by realizing the same gain (loss) over a shorter fiber using a higher pump power. Actually the group index change will vary from the $10^{-3}$ range for kilometre-long fibers to the unity range for meter-long fibers. When such a short fiber is used, the main issue is to raise the pump power to about 10 Watts to obtain $\Delta n_{g}$ 's larger than unity. This can be achieved by forming a pulse train with the pump wave, so that the full gain is available in the erbium-doped fiber amplifier (EDFA) while the average output power is kept below the saturation power.

We used a 2-m section of a standard fiber with a $v_{B}$ of $10.907 \mathrm{GHz}$, while the rest of the fibers used in the setup showed $v_{B}$ 's that were different enough from that of the sample (100 MHz smaller), so that it can be certified that the interaction only takes place in the test fiber. A DFB laser diode operating at $1552 \mathrm{~nm}$ was used as a light source, and its output was launched to an electro-optic modulator (EOM) to create two first-order sidebands. The carrier wave was suppressed by controlling the DC bias voltage delivered into the EOM with a feedback circuit [5]. The frequency difference between the two sidebands was set to the $v_{B}$ of the fiber.

In order to measure the effect of Brillouin gain, the lower sideband (Stokes wave) was reflected by a narrow band fiber Bragg grating and optically gated to be used as a probe pulse. The upper sideband (anti-Stokes wave) was also gated to create a Brillouin pump pulse and its instantaneous power was amplified to $\sim 10 \mathrm{~W}$ using a couple of high power EDFAs. As fast optical gates, another EOM was used in both cases, resulting in clean pulses with sharp rising and trailing edges. We used a variable attenuator to control the amplitude of the strong pump pulse, and adjust the timing of pump and probe pulses to make them overlap during the propagation in the test fiber. In addition, the duration of the pump pulse was made longer than the whole propagation time of the probe pulse in the test fiber, so that the probe pulse sees a constant pump power while propagating throughout the fiber.

The time delay of the probe pulse was measured using a digital oscilloscope for different values of Brillouin gain by varying the pump amplitude from zero to $\sim 10 \mathrm{~W}$. In the mean time, the output amplitude of the probe pulse on the detector was kept constant using another variable attenuator to avoid a possible time biasing from an amplitudedependent time response of the detector. In the case of Brillouin loss experiment, we swapped the roles of the two sidebands.

\section{Results}

The gain was swept from $-12 \mathrm{~dB}$ to $21 \mathrm{~dB}$ with $3 \mathrm{~dB}$ steps, and the pulse width (FWHM) of the probe was set to $40 \mathrm{~ns}$. We used a 120-ns square pulse for the Brillouin pump so that the probe pulse sees a constant gain/loss during the interaction. It is remarkable that the maximum observed delay and advancement in this $2-\mathrm{m}$ fiber $(\sim 10 \mathrm{~ns})$ are fully comparable to those obtained in several kilometre-long fibers [1]. 


\section{WC3}

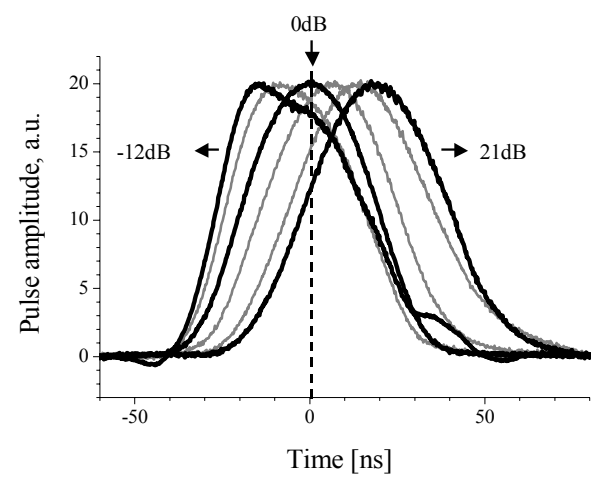

(a)

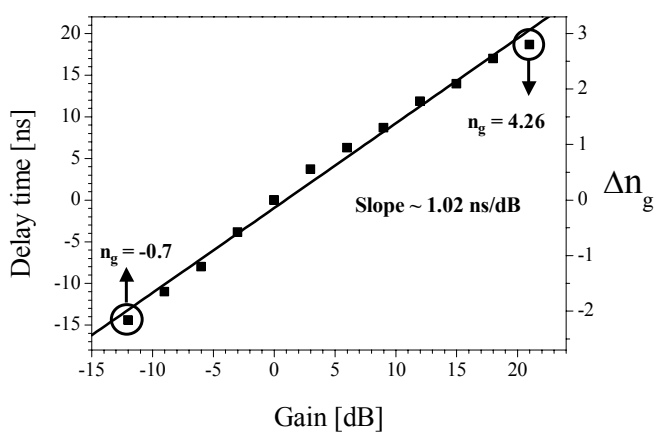

(b)

Fig. 2: (a) Pulse delaying and advancement in a 2 meter fiber using SBS (b) corresponding group delay changes.

Fig. 2(a) shows time waveforms of pulses experiencing different gains and losses through stimulated Brillouin scattering in the short fibre. The observed delays are fully comparable in this $2 \mathrm{~m}$ sample to those obtained along several kilometres of fibre. By measuring the pulse waveform under normal condition $(0 \mathrm{~dB}$ gain $)$ and subtracting the $9.8 \mathrm{~ns}$ normal propagation delay in the fibre sample, we could retrieve the time position of the pulse at the fibre input and experimentally demonstrate that the pulse peak is transmitted before entering the fibre, confirming the presence of a negative group velocity. Fig. 2(b) shows the pulse peak position as a function of the gain (loss) experienced by the signal and the equivalent group index change. This index could be increased continuously from 1.46 in normal conditions to 4.26 under high Brillouin gain, and lowered to -0.7 under high Brillouin loss, that is a highly superluminal propagation. In other words the group velocity could be changed continuously from $70^{\prime} 500 \mathrm{~km} / \mathrm{s}$ to infinite and then to $-428^{\prime} 000 \mathrm{~km} / \mathrm{s}$ in the fibre sample $\left(\sim 205^{\prime} 000 \mathrm{~km} / \mathrm{s}\right.$ in normal conditions), leading to the impressive delays from $-14.4 \mathrm{~ns}$ to $+18.6 \mathrm{~ns}$ in only 2 meter of fibre. It means that the fibre effective length can be continuously changed from $-3 \mathrm{~m}$ to $3.8 \mathrm{~m}$.

It must be pointed out that a group velocity faster than the vacuum light velocity $c$ or even negative does not break the famous principles resulting from causality and relativity, as demonstrated by Brillouin himself [6]. The fact that the spectral transition is narrowband prevents all frequency components from undergoing the extreme group velocity and the information velocity thus cannot exceed $c$. This leads to a considerable distortion of the pulse in the case of fast light (starting and ending points at normal light velocity in the medium, peak point at modified group velocity), resulting in a steeper leading edge and a longer trailing edge. This can be clearly observed in Fig. 2(a) in the case of strong pulse advancement.

\section{Conclusion}

We have demonstrated a wide-range control of the group velocity of light signals in optical fibers using stimulated Brillouin scattering. With a simple tabletop configuration in normal environmental condition, we have achieved nearly all results obtained using atomic transitions, from slow light with a group velocity as slow as $71,000 \mathrm{~km} / \mathrm{s}$ to superluminal propagation and even negative group velocity. We expect this work could be the platform for the development of a wide range of applications.

\section{References}

[1] K. Y. Song, M. G. Herráez, and L. Thévenaz, "Observation of pulse delaying and advancement in optical fibers using stimulated Brillouin scattering," Opt. Express 13, 82-88 (2005), http://www.opticsexpress.org/abstract.cfm?URI=OPEX-13-1-82.

[2] Y. Okawachi, M.S. Bigelow, J. E. Sharping, Z. Zhu, A. Schweinsberg, D.J. Gauthier, R.W. Boyd, and A.L. Gaeta, "Tunable All-Optical Delays via Brillouin Slow Light in an Optical Fiber", Phys. Rev. Lett. 94153902 (2005).

[3] K. Y. Song, M. G. Herráez, and L. Thévenaz, "Long optically-controlled delays in optical fibers" Opt. Lett. to appear (2005)

[4] R. W. Boyd and D. J. Gauthier, "'Slow' and 'Fast' Light," Ch. 6 in Progress in Optics 43, E. Wolf, Ed. (Elsevier, Amsterdam, 2002 ), 497530.

[5] M. Niklès, L. Thévenaz, and Ph. Robert, "Brillouin gain spectrum characterization in single-mode optical fibers," J. Lightwave Technol., LT-15, 1842-1851 (1997).

[6] L. Brillouin, "Wave propagation and Group Velocity," (Academic Press, New York, 1960). 